\section{Integrative Medicine \\ International}

\title{
Does the Primo Vascular System Originate from the Polar Body
}

\author{
Majid Avijgan a Mahtab Avijgan ${ }^{b}$ \\ ${ }^{a}$ Department of Traditional Persian-Iranian Medicine, Isfahan University of Medical \\ Sciences, Isfahan, Iran; ${ }^{b}$ University of Newcastle, Newcastle, N.S.W., Australia
}

\section{Key Words}

Embryo $\cdot$ Embryonic development $\cdot$ Polar body $\cdot$ Primo vascular system

\begin{abstract}
Background: Polar bodies degenerate on the first day of embryonic life, and there is no known or specific role for them in the human embryo yet. However, it is not logical to have useless cells. Our previous reports indicated a role for the primo vascular system (PVS) in human beings. There is a possible link between polar bodies and the PVS in embryonic life. Methods: The following databases were searched for peer-reviewed articles in English: Cochrane Collaboration, PubMed, MEDLINE, CINAHL, AMED, Age line, and Social Services Abstracts. Our search included the following key words individually or in combination: alternative medicine(s), integrated medicine, holistic care, complementary alternative medicine, embryology, traditional Chinese medicine, traditional medicine, PVS, Bong Han duct in relation to polar bodies. Results: There are no reports relating polar bodies to our keywords. Conclusion: The PVS has been suggested to be responsible for embryonic development. This system contains some pluripotent cells and proteins, some of which are not even present in the blood circulation in chicken embryos. The system becomes only visible after staining with trypan blue. Accordingly, this report tries to present the PVS as the ruler of embryonic cell division and development, which regulates all complicated events during that period of life. To the best of our knowledge, this is the first report suggesting that embryonic development is controlled via the PVS, which originates from polar bodies.
\end{abstract}

(C) 2014 S. Karger AG, Basel

\section{Introduction}

It seems that the human embryogenic development is not a simple process of cell division and development, still it must be based on laws and regularity that can control and coordinate this complicated process. In 1996, using human embryos for research purposes was banned. This total ban was revised in 2000 regarding the issues of researching damage and destruction

Prof. Dr. Majid Avijgan

PO Box 795

Alzahra Hospital, Soffeh St, Isfahan (Iran)

E-Mail avijgan@yahoo.com
Miss Mahtab Avijgan

University of Newcastle

Newcastle, NSW 2300 (Australia)

E-Mail mahtab.avijgan@gmail.com 
Table 1. The steps of polar body formation

\begin{tabular}{ll}
\hline Steps in the cell division & Fmn2 $\rightarrow$ Cdc42 activation and Src family kinase proteins \\
\hline Effect on the next step & $\begin{array}{l}\text { Fmn2 produces dynamic actin meshwork and co-localization } \\
\text { Cdc42 activation leads to membrane protrusion } \\
\text { Src leads to asymmetric cytokinesis }\end{array}$ \\
\hline $\begin{array}{l}\text { Process of polar body } \\
\text { formation }\end{array}$ & $\begin{array}{l}\text { Spindle during meiosis } \rightarrow \text { membrane protrusion } \rightarrow \text { activity polar body formation in the process } \\
\text { precise temporal restraints of asymmetric cytokinesis } \rightarrow \text { division polar body formation }\end{array}$ \\
\hline
\end{tabular}

in human embryos [1]. This presents some limitation when studying the complicated developmental steps of human conception.

Recently, there has been a report on findings in chicken embryos which aimed to describe these complex processes. It introduces the so-called primo vascular system (PVS), which is a newly discovered circulatory system that can regulate many embryonic developmental processes [2]. The basic concept suggests that the PVS is a specific organization existing in many animals and even human beings [3]. So far, there are no reports on the PVS in human beings, requiring it to be proven. In our previous report [4], we suggested that this system may be the base of embryonic development and growth. How, where and when is the PVS produced? Here we try to answer these questions and many others.

\section{What Is the Polar Body?}

The first report on polar bodies was written in 1824 by Carus; however, their characteristics were only reported many years later. They would get confused with an expelled yolk mass and later with the directional body which determines the place where maturation division begins [5]. In 1881, some 50 years after that first report, several other roles had been indicated for polar bodies such as 'aborted ova', that of a rapid 'tester' [6] and tool in pre-implantation genetic diagnoses [7] or as a metric for the oocyte in tests for embryonic viability [8].

Polar bodies are typically formed by asymmetric cytokinesis: this leaves the ovum's sister with relatively little cytoplasm, and thus they simply degenerate in most organisms [5]. As shown in table 1, formin-2 (Fmn2) is a specific protein that is co-localized with the spindle during meiosis and is involved in the formation of a dynamic actin meshwork [9]. After an asymmetric positioning of the meiotic spindle and by Cdc42 activation, the membrane protrusion activity probably operates through an actin meshwork mechanism [10] (table 1). It seems that in some animals' oocytes, such as the mouse's, the actin-nucleating protein of Fmn2, an important protein in cytokinesis, is required for spindle migration [9]. Cdc42 promotes the membrane protrusion, an essential change needed for the formation of polar bodies. This function of Cdc42 appears to be unique to female meiosis, so inhibiting Cdc42 was shown to be sufficient to prevent the extrusion of the second polar body. All these processes are suggesting an essential function for this protein [10]. Src family kinase proteins may be involved in maintaining the precise temporal restraints of asymmetric division, regulating the size of polar bodies by controlling filamentous actin turnover during the formation and ingression of the cleavage furrow [11] (table 1). Thus, the polar body is formed by a complicated and cascaded process.

In human oocytes, polar body apoptosis takes place 17-24 $\mathrm{h}$ after its formation, and the resulting fragments remain entrapped within the zona pellucida [12]. In many other organisms, however, polar bodies are not dispensable. Animals, for example, use multiple mechanisms of parthenogenesis, most of which are dependent upon polar bodies. The nucleus of the polar body divides without cytokinesis and probably migrates as extraembryonic tissue surrounding 
the dividing blastomeres. This enables the embryo to feed on the host's body and may also defend the embryo against the host's immune system [5].

The polar body can be fertilized and results in the formation of a human embryo [13]. This finding confirms that polar bodies possess the complete genetic information needed for the formation of a new embryo. In some animal species, polar bodies organize the embryonic development in separate steps. For example in Copidosoma floridanum, polar bodies persist for about 10 days, and they seem to dominate most aspects of embryonic development [5]. Polar bodies can play a role like endosperm in some other animals [14]. However, a recent study showed that no twin pairs can result from the fertilization of the second (haploid) polar body [15].

These various traits of polar bodies lead to several conclusions. First of all, polar bodies can be extraembryonic tissue which is a source for embryonic nourishment [5]. Secondly, they can split up and (after the second division) they will degenerate (disappear) within 17-24 h after their formation [12]. Thirdly, after the first division, they have the capacity of generating into a new embryo [9], but not after the second division [15]. And finally, polar bodies dominate most aspects of embryonic development [5].

This leads to some questions. Can the PVS originate from polar bodies? Do the disappearing polar bodies rather transform into the PVS? Since the PVS is generally invisible, does its recognition depend on the method of staining (e.g. trypan blue staining)? Is the PVS a system or network which includes all aspects of the entire human body? And as a bodyrepairing 'reservoir' (including stem cells, SCs), can the PVS originate from polar bodies which have the potential to be fertilized?

Based on these thoughts, we hypothesize that the PVS - as an invisible system in the human body - must have originated from polar bodies. If, on the one hand, polar bodies have an important role in primitive beings and, on the other hand, the PVS is a primitive system of circulation, then the two must have a mutual relationship. Given this theory and based on the best of our knowledge, this is the first time to claim that the PVS originates from polar bodies. In this report, we try to track the formation of polar bodies, their degeneration, the probable formation of the PVS and its role in human embryos.

\section{Materials}

The literature was reviewed looking for 7 key words/themes: the primo vascular system, PVS, organogenesis, embryogenesis, traditional Chinese medicine (TCM), traditional medicine (TM) and polar bodies.

\section{Search Strategy}

The following databases were searched for peer-reviewed articles in English: Cochrane Collaboration, PubMed, MEDLINE, CINAHL, AMED, Age line, and Social Services Abstracts. Our search included the following key words individually or in combination: alternative medicine(s), integrated medicine, holistic care, complementary alternative medicine, embryology, traditional Chinese medicine (TCM), traditional medicine (TM), primo vascular system (PVS), Bong Han duct in relation to polar bodies. There are no reports explaining the implications of the PVS in embryogenesis.

Our hypothesis is necessary to be approved by the following developmental periods of human embryos, accompanied by pictures or figures during organogenesis or embryonic life. The PVS is a broad network in the human body, it is similar to an invisible structure, connecting all body organs to each other to produce a whole. This system is distributed in connective tissue, surface organs, internal organs, intravascular, extravascular, pre-neuronal and intraventricular brain [4]. 


\section{Results and Discussion}

'Ontogeny recapitulates phylogeny' summarizes in evolutionary terms that if a structure pre-dates another structure, then it often appears before the others in an embryo [16]. But even if this law is correct, it is not reasonable that the complicated and cascaded steps of fetal development are unintentional or unplanned. It seems that the PVS hypothesis can clear the details of Gould's hypothesis that 'ontogeny recapitulates phylogeny'. In other words, 'ontogeny recapitulates phylogeny' or the biogenetic law can be better explained by the PVS hypothesis or the PVS law.

\section{The PVS Hypothesis or the PVS Law}

The PVS is a circulatory system which has only recently been discovered in many animals and even in human beings [3]. It consists of ducts, corpuscles and granules floating in those ducts. A granule (sanal) is composed of one sanalsome (containing a large amount of DNA) and sanalplasm (containing RNA). The granule's composition of bases and nucleotides is the same as that of a normal cell. A granule (sanal) can grow into a cell, and reversely a cell can become a granule (sanal) in the 'sanalization process'. Each granule (sanal) contains the same amount of DNA as a chromosome. During the sanalization process, the number of sanalsomes is the same as that of the chromosomes [16]. (One cell transforms into 46 sanalsomes.) The primo micro cell (PMC, sanal or granule) is produced by a cell's sanalization process. PMCs move into the primo vessels and their nucleus-like structure can grow, mature and migrate to local tissues to produce new tissues [17]. All human tissues and cells have been suggested to be linked to the PVS [4]. Nucleic acid granules (sanals) have been found to play a crucial role in the physiological functions of the PVS, especially in the regeneration of dead cells. In the PVS, 70 different proteins have been discovered in the liquid and 270 proteins in the vessels, many of them are not normally found in the blood, or in lymph or blood vessels [18].

Granules (sanals or small cells) or PMCs actively proliferate and then migrate through the PVS into different tissues to differentiate into adult SCs. A rare proportion of very small embryonic-like SCs (VSELSCs) in the bone marrow and other adult tissues share several marker characteristics with pluripotent SCs (PSCs), and these characteristics are similar to those of epiblast/germ layer line-derived SCs [19]. In other words, VSELSCs are mediator cells which have been transformed from PSCs and will be transformed into epiblast/germ layer line-derived SCs and finally adult SCs:

PMCs $\rightarrow$ PSCs $\rightarrow$ VSELSCs $\rightarrow$ epiblast/germ layer line-derived SCs $\rightarrow$ adult SCs.

PMCs grow rapidly and will be transformed into PSCs or young undifferentiated SCs. A type of PMCs are VSELSCs [20]. However, it seems that epiblast/germ layer line-derived SCs are derived from undifferentiated SCs (PSCs) [19], thus VSELSCs are specifically suggested as deposited cells in organs during organogenesis [19].

Which mechanism(s) controls the process of organogenesis (resulting from epiblast/ germ layer line-derived SCs during gastrulation)? PMCs or VSELSCs (and/or the PVS) or other mechanisms? The answer can be: the PVS through VSELSCs (PMCs). In this case, VSELSCs (PMCs) firstly control organogenesis and secondly play the role of a messenger as a deposited cell in any organ during organogenesis and gastrulation.

Having several similar marker characteristics hints at the relationship between VSELSCs (in the PVS), PSCs (undifferentiated SCs) and epiblast cells in embryos, i.e. one could be the precursor of the other. But which one is first? It is the old story of the egg and the chicken. It could be postulated that the sanal cells (PMCs-VSELSCs) in the PVS of the embryo are probably the precursor and/or transformer of the inner cell mass into epiblast cells, which is the origin of the three embryonic layers. This hypothesis also assumes that, in embryonic life and the 
Fig. 1. Zona pellucida containing the PVS. The PVS can be found between the developing zygote and the zona pellucida.

\begin{tabular}{l|l}
\hline DOI: 10.1159/000368650 & $\begin{array}{l}\text { C 2014 S. Karger AG, Basel } \\
\text { www.karger.com/imi }\end{array}$ \\
\hline Avijgan and Avijgan: Does the Primo Vascular System Originate from the Polar Body
\end{tabular}

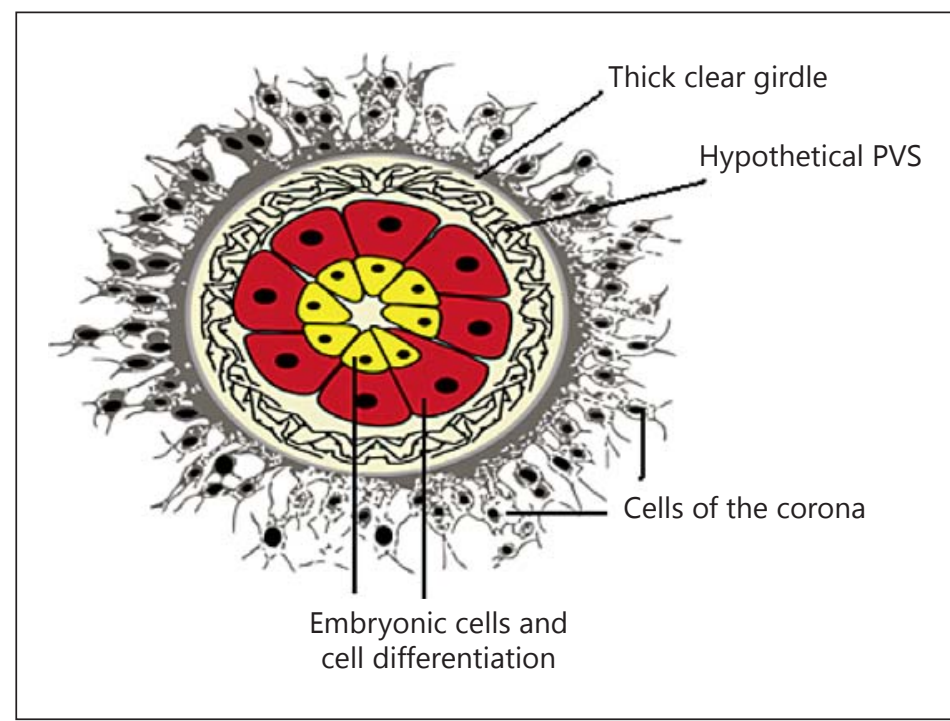

Fig. 2. The mono-cell zygote showing the fertilizing zygote, polar body (the hypothetical pioneer of the PVS) and the zona pellucida, as a shell for the zygote.

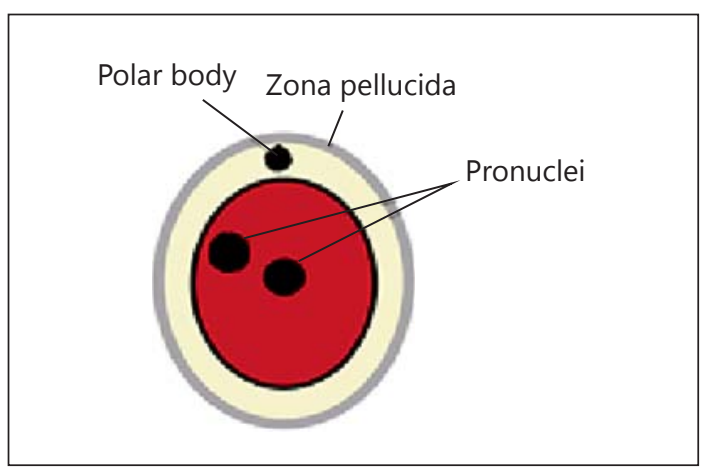

developmental period of an embryo, the PVS rules the differentiation of epiblast cells into the three (next) layers ectoderm, mesoderm and endoderm of an embryo. But because epiblast cells are the source or original cells, the sanal cells which can be the origin of PMCs (VSELSCs in the PVS) or epiblast cells (in the embryo) are deposited in each organ during gastrulation and organogenesis and may serve as reserve pool of quiescent PSCs [19].

The most probable place to find the PVS is the zona pellucida. During zona hatching, the PVS matures into a developed system and may sustain both itself and also a new fertilized but developing zygote (fig. 1). This supportive role has been reported for animals, too [5]. This kind of system, which may be in the zona pellucida and preserve the union of morula, has been suggested to be the PVS or proto PVS, similar to what has been reported in chicken embryos [2,3].

One idea that has been mentioned is that the PVS appears before or simultaneous to the fertilization of the ovum by the sperm. Based on this hypothesis, such a mechanism can inhibit the entrance of a second sperm into the (now fertilized) ovum. In other words, this has been suggested to be the first role of the PVS in the new life of a human: to keep the package of zygote. If this proves to be true, further studies should be conducted searching for basophilic granules in the ovum and sperm. It needs a special staining of the sperm and/or ovum to search for any presence of the PVS. In support of this later hypothesis, the first cell formation after fertilization shows two pronuclei and one polar body. This polar body can transform into the PVS and control the mysterious mechanisms of fetal development (fig. 2). The domi- 


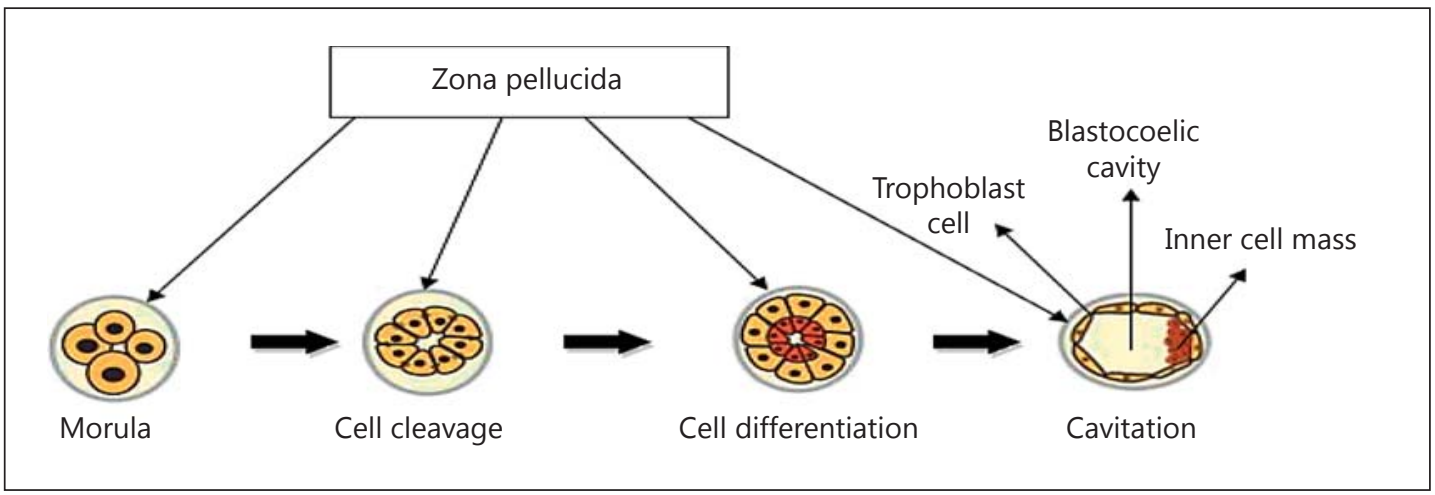

Fig. 3. Morula, cell cleavage, cell differentiation and cavity formation of the fertilized ovum.

nating role of polar bodies in animal embryonic development has been reported in a previous study, too [5]. The results of another study support 'a long-standing belief that chromosomes ejected from the first polar body have the same genetic potential as those remaining in the oocyte after the first meiotic division. The chromosomes in the second polar body are known to have full potential to participate in normal embryonic development' $[20,21]$. So the genetic code of polar bodies is similar to that of embryonic cells. A previous study reported of potentially embryonic fertilization, too [13].

The supportive and dominating role of polar bodies [5] and their potential to be fertilized [13] can lead to another hypothesis: polar bodies could be the pioneers for the PVS. After fertilization, the polar bodies form the primitive PVS and other cell(s) transfer the genetic codes to the zygote. In this way, the polar bodies control the development of the growing zygote to mature into a well-developed human being. This transformation into the network of pre-PVS to proto-PVS to PVS [2] happens in the space between dividing zygote and zona pellucida, during the first week of life and before the zona hatching occurs.

\section{Embryonic Period [22]}

After fertilization, the embryonic life starts and continues until the end of gestational week 8 (in humans). The dividing cells form a mass and then a ball of cells called the morula. These early divisions produce smaller cells. However, through further divisions, a small cavity between the cells is produced. The divided cells are interconnected by tight cell junctions after day 3 of fertilization. The daughter cells are blastocyst and include lining cells as trophoblast, an inner cell mass and a blastocyst cavity (blastocoelic cavity; fig. 3). Similar to our hypothesis, in the early hours of chicken egg fertilization (which are comparable to the early days of human zygotes), the appearance of the PVS in the vitelline membrane of the chicken egg has been reported [2] (fig. 4). Our PVS hypothesis has been based on this discovery. The zona pellucida has been suggested to have the role of thick albumin (and containing the PVS) and surrounds the yolk sac of the chicken egg. If the PVS is a part of the conception product structure, then it can be responsible for early cell differentiation, junction and the cavitation process. This could be the answer to the main question of what is responsible for these complicated developmental processes in a fetus (fig. 5). The PVS has been located in the thick albumin layer and outer area of chicken embryos (fig. 4). Localizing the PVS would be a very important step and phase in human embryology research, which has been taken for granted for so long. In a previous study, it has been reported that polar bodies are transformed into extraembryonic tissue [5]; based on our theory, the PVS is presented as extraembryonic tissue (located between the zygote and the zona pellucida). This statement might suggest that the PVS originates from polar bodies. 


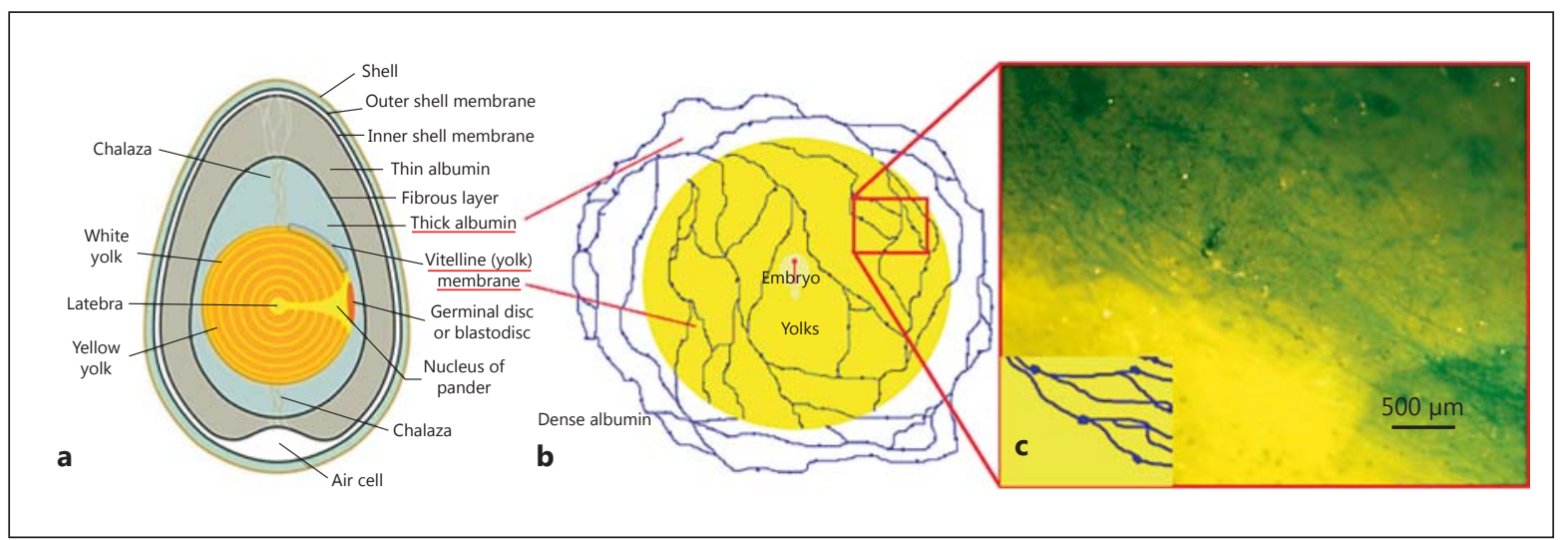

Fig. 4. a Longitudinal cross section of a chicken egg (after Lillie). b Trypan blue staining on the vitelline reveals a complicated network on the vitelline and white thick albumin and some dots which seem to be DNAcontaining bodies. c Magnified view of the square in $\mathbf{b}$. The curves and dots might be related to the PVS. The egg has been incubated for 16-24 h, corresponding to stages 4-7 according to Hamburger and Hamilton's criteria. Reprinted with permission from Dr. Kwang-Sup Soh [2].

In the first week of embryonic life (after fertilization), the products of conception include the predecessor of the embryo (in the form of a blastocoelic cavity; fig. 1); its membranes (postulated the PVS) are surrounded by the zona pellucida. Before the implantation into the uterine wall, the zygote has to come out of the zona pellucida [23]. During implantation, the embryo is embedded in the uterine (endometrium). This lysis of the zona pellucida is called the zona hatching (fig. 6). According to this hypothesis, the PVS which is in the zona pellucida (as the extraembryonic PVS) controls all developmental processes of the products of conception, including trophoblast, inner cell mass differentiation, zona hatching and differentiation (fig. 5, 6). The dominating role of polar bodies for all aspects of embryonic development has been reported in a previous study [5], similar to the role that is postulated for the PVS in our hypothesis.

\section{Inner Cell Mass Differentiation}

It has been speculated that the extraembryonic PVS surrounds the epiblast (primitive ectoderm; fig. 6). The epiblast, now called primitive ectoderm, performs gastrulation, approximately on day 16 (week 3) after fertilization [24]. 'It is not birth, marriage, or death, but gastrulation, which is truly the most important time in your life.' The terms gastrulation and gastrula were coined in 1872 [25].

\section{Gastrulation}

This is an important phase during which the single-layered embryo (blastula) is rearranged into the ectoderm, mesoderm and endoderm [24]. It has been suggested that the extraembryonic PVS surrounded by the zona pellucida and extraembryonic membranes (fig. 5,6 ) could be responsible for all these automatic phenomena in human embryos. This subject needs to be tested and assessed in laboratories.

\section{Loss of Symmetry}

Up to day 16, the embryo has a symmetrical presentation. At the beginning of gastrulation, two symmetry-braking events must take place in the embryo: one along the proximaldistal axis (the placenta at the proximal end and the epiblast at the distal end) [26] and one 


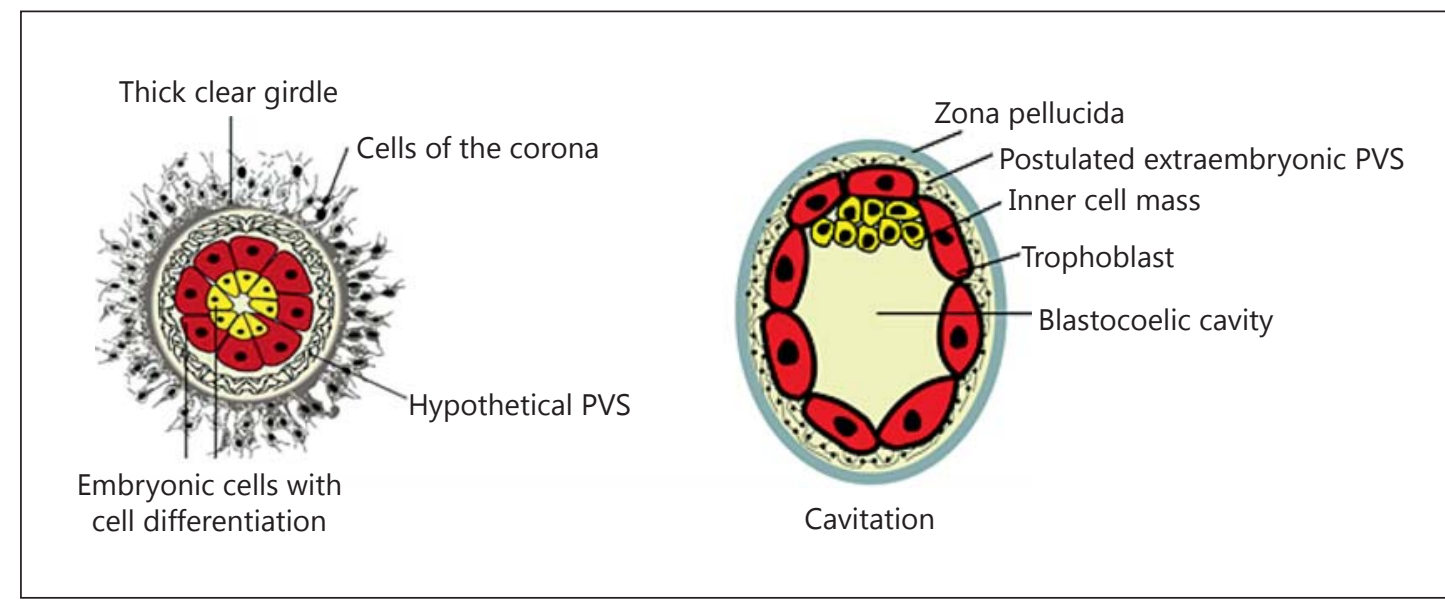

Fig. 5. An embryo at the cavity-forming stage. The schematic view of the embryonic cells includes the inner mass cells and the blastocells that are surrounded by the hypothetical PVS. The outer-most layer is the zona pellucida. Cells of the corona are depicted in the right image but not in the left image.

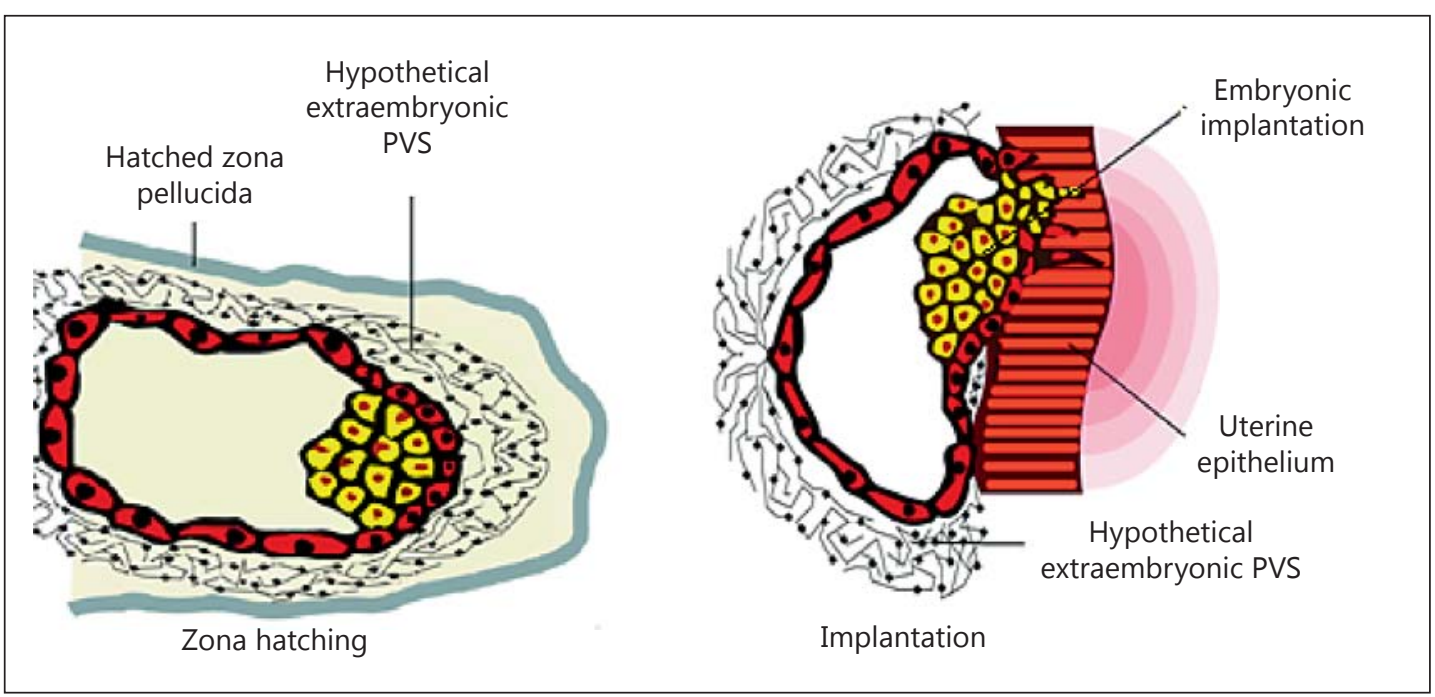

Fig. 6. Zona hatching and implantation of the fertilized egg showing the embryo (blastocyst) and the hypothetical PVS.

along the anterior-posterior axis. Here, the epiblast is on the posterior side of the embryo where the ingression will start [27]. The primitive streak has very important roles in the embryonic development. It establishes bilateral symmetry, the place of gastrulation and it also stimulates the germ layer formation. This process involves the ingression of mesodermic and endodermic progenitors and their migration to their ultimate position [28, 29], where they will differentiate into the three germ layers [30]. A fine system, such as the extraembryonic PVS, could be suggested which controls and defines the time and place for the braking events (in both the proximal-distal and anterior-posterior direction), primitive streak, mesoderm induction and gastrulation. Furthermore, the left-right, front-back and top-bottom direction of the embryo must be 'allocated'. This suggested role of the dominating PVS (polar bodies) is similar to that of previous reports [5]. 

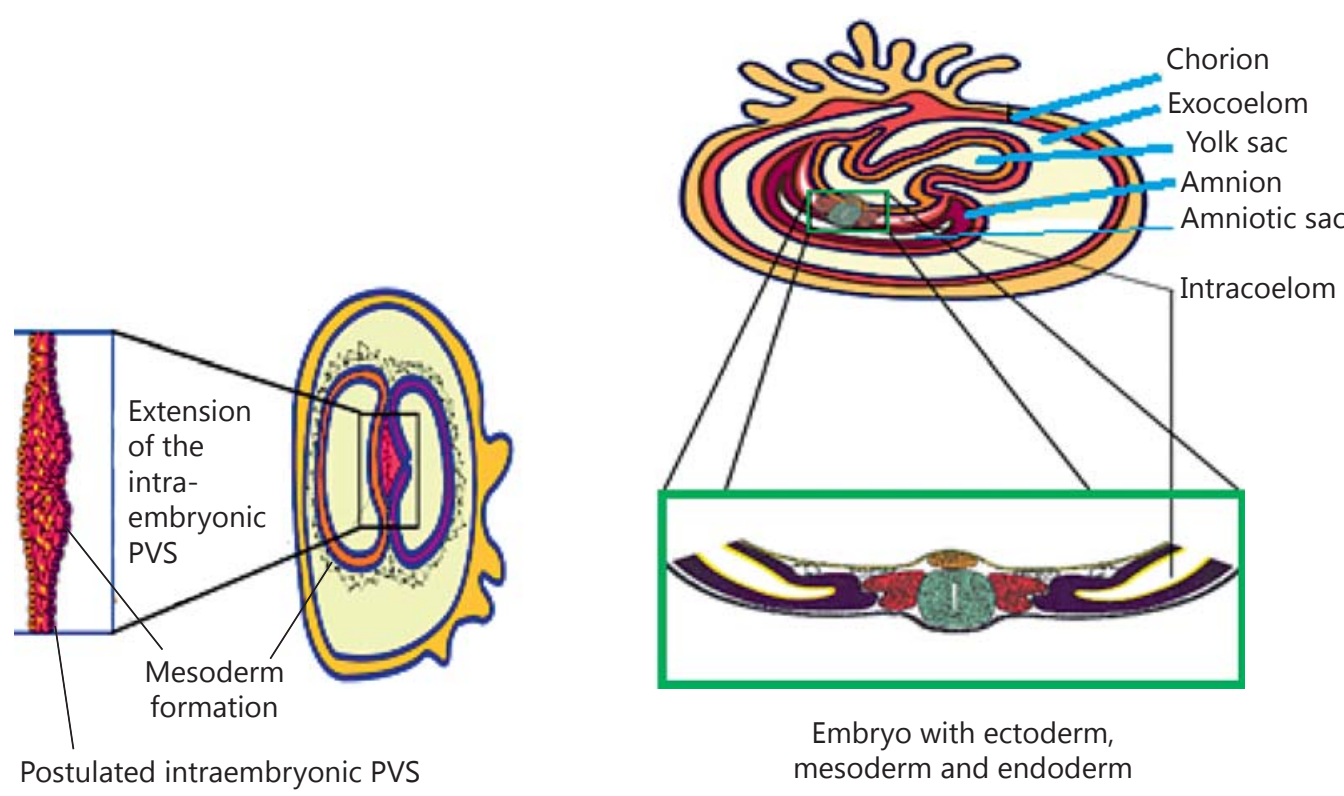

Fig. 7. Mesoderm formation and spreading (including intracoelom?).

As the embryo continues to grow/develop, that side of the ovum on which the sperm has entered becomes the belly, front or Yin side, which is subdivided into right and left by the Ren meridian. The opposite side of the ovum becomes the back or Yang side, which is subdivided by the Du meridian [31]. Animal study have suggested that the left-right axial structures in the early embryonic development are established using several proteins. A complex and cascaded process [26, 27, 32-35].

It has been suggested that some part of the extraembryonic PVS accompanied by the primitive streak will ingress into the embryo (during gastrulation) and form the intraembryonic PVS (fig. 7).

The epithelial to mesoderm transition is a process which leads to the formation of new cells, which - through the primitive streak - will ingress and spread out to form a new layer of cells or join existing layers (ectoderm, mesoderm and endoderm) [36]. The ectoderm gives rise to the skin and central nervous system. The endoderm produces the endothelial cells of the digestive and respiratory systems and some organs associated with the digestive system, such as the liver and pancreas [26]. Mesenchymal cells stem from the mesoderm. They can migrate easily and are characterized morphologically by a prominent matrix containing a loose aggregate of reticular fibular (collagen?) and unspecialized SCs [24]. The mesoderm seems to be the most important layer of the embryo. It gives rise to all major organs of the body.

\section{Conclusion}

This report indicates for the first time a transformation of polar bodies into the PVS as an extraembryonic tissue; the PVS having a supportive, dominating and controlling role in all steps of embryonic development. It seems to have been missed or ignored because it is not 
visible by usual staining. Trypan blue staining and some other specific stainings can make it visible for a short time. During the embryonic life (after the third week of gestation), the embryo undergoes major changes like the primitive streak, loss of symmetry, gastrulation and the formation and differentiation of endoderm, mesoderm and ectoderm. The extraembryonic PVS has been suggested to have an important and key role in this.

The extraembryonic PVS has been assumed to extend around the embryo and be responsible for the generation of structures like the chorion and yolk sac. In the gastrulation process, it has been suggested that some part of this PVS will ingress into the embryo and form the intraembryonic PVS (fig. 7). While the intraembryonic PVS is spreading amidst the mesoderm, this system will be split into two parts during the delamination of the mesoderm into two layers (fig. 7). One part is in the inner surface of the splanchnic mesoderm, the other part is in the inner surface of the somatic mesoderm. The somatic mesoderm together with the ectoderm stimulates the production and generation of organs like the amnion during the embryonic life and also the skin. The splanchnic mesoderm together with the endoderm, one the other hand, stimulates the generation of the mucosal cells of the liver and organs like the lung and those of the gastrointestinal tract. In fact, the mesoderm has a great responsibility by inducing some other internal organs and structures like the heart, kidney, spleen and connective tissue (the PVS hypothesis; fig. 7).

\section{Acknowledgement}

We thank Mrs. Fatemeh Abdollahi for her kindness and helpful editing of this article.

\section{References}

1 Committee on Pediatric Research and Committee on Bioethics: Embryonic stem cell research. Pediatrics 2001; 108:813-816.

$\rightarrow 2$ Lee SY, Lee BC, Soh KS, Jhon GJ: Development of the putative primo vascular system before the formation of vitelline vessel in chicken embryo; in Soh K-S, Kang KA, Harrison DK (eds): The Primo Vascular System: Its Role in Cancer and Regeneration. New York, Springer, 2011, pp 77-82.

-3 An P, Su Z, Luo H, Soh K-S: Primo vessels in the mesentery of nude mice; in Soh K-S, Kang KA, Harrison DK: The Primo Vascular System: Its Role in Cancer and Regeneration. New York, Springer, 2011, pp 101-105.

4 Avijgan M, Avijgan M: Can the primo vascular system (Bong Han duct system) be a basic concept for Qi production? Int J Integr Med 2013;1:20.

5 Schmereler S, Wessel G: Polar bodies - more a lack of understanding than a lack of respect. Mol Reprod Dev 2011;78:3-8.

6 Wella D, Hillier SG: Polar bodies: their biological mystery and clinical meaning. Mol Hum Reprod 2011;17: 273-274.

7 Kuliev A, Rechitsky S: Polar body based preimplantation genetic diagnosis for Mendelian disorders. Mol Hum Reprod 2011;17:275-285.

-8 Klatsky PC, Wessel GM, Carson SA: Detection and quantification of mRNA in single human polar bodies: a minimally invasive test of gene expression during oogenesis. Mol Hum Reprod 2010;16:938-943.

-9 Kwon S, Shin H, Lim HJ: Dynamic interaction of forming proteins and cytoskeleton in mouse oocytes during meiotic maturation. Mol Hum Reprod 2011;17:317-327.

10 Leblanc J, Zhang X, McKee D, Wang ZB, Li R, Ma C, Sun QY, Liu XJ: The small GTPase Cdc42 promotes membrane protrusion during polar body emission via ARP2-nucleated actin polymerization. Mol Hum Reprod 2011;17: 305-316.

11 Levi M, Kaplan-Kraicer R, Shalgi R: Regulation of division in mammalian oocytes: implications for polar body formation. Mol Hum Reprod 2011;17:328-334.

12 Longo FJ: Fertilization. New York, Chapman and Hall, 1997.

13 Bieber FR, Nance WE, Morton CC, Brown JA, Redwine FO, Jordan RL, Mohanakumar T: Genetic studies of an acardiac monster: evidence of polar body twinning in man. Science 1981;213:775-777.

-14 Queller DC: Kin selection and conflict in seed maturation. J Theor Biol 1983;100:153-172.

15 Machin G: Non-identical monozygotic twins, intermediate twin types, zygosity testing, and the nonrandom nature of monozygotic twinning: a review. Am J Med Genet 2009;151C:110-127. 
16 Gould SJ: Ontogeny and Phylogeny. Cambridge, Belknap, 1977, p 206.

-17 Lee JS: Recollection of early research on primo vascular system; ultimate implication of Bong Han theory; in Soh K-S, Kang KA, Harrison DK (eds): The Primo Vascular System: Its Role in Cancer and Regeneration. New York, Springer, 2011, pp 23-24.

18 Vadyanoy V: Characterization of primo nodes and vessels by Hugh resolution light microscopy; in Soh K-S, Kang KA, Harrison DK (eds): The Primo Vascular System: Its Role in Cancer and Regeneration New York, Springer, 2011, pp 83-94.

19 Ogay V, Soh K-S: Identification and characterization of small stem-like cells in the primo vascular system of adult animals; in Soh K-S, Kang KA, Harrison DK (eds): The Primo Vascular System: Its Role in Cancer and Regeneration New York, Springer, 2011, pp 149-154.

20 Wakayama T, Yanagimachi R: The first polar body can be used for the reproduction of normal offspring in mice. Biol Reprod 1998;59:100-104.

-21 Lee M, Munne S: Pregnancy after polar body biopsy and freezing and thawing of human embryos. Fertil Steril 2000;73:645-647.

22 Wikipedia: Human embryogenesis. 2014. http://en.wikipedia.org/wiki/Human_embryogenesis (accessed December 2012).

23 IVF-infertility: IVF techniques: provides information on assisted hatching, a technique to improve the implantation rate. 2011. http://www.ivf-infertility.com/ivf/hatching.php (accessed December 2012).

-24 Mundlos S: Gene action: developmental genetics; in Speicher MR, Motulsky AG, Antonarakis SE (eds): Vogel and Motulsky's Human Genetics: Problems and Approaches, ed 4. Berlin, Springer, 2010, pp 417-450.

25 Wolpert L, Tickle C: Principles of Development. Oxford, Oxford University Press, 2011. ISBN 978-0-19955428-7.

-26 Arnold SJ, Robertson EJ: Making a commitment: cell lineage allocation and axis patterning in the early mouse embryo. Nat Rev Mol Cell Biol 2009;10:91-103.

27 Tam PL, Behringer RR: Mouse gastrulation: the formation of a mammalian body plan. Mech Dev 1997;68:3-25.

28 Downs KM: The enigmatic primitive streak: prevailing notions and challenges concerning the body axis of mammals. Bioessays 2009;31:892-902.

29 Chuai M, Weijer CJ: The mechanisms underlying primitive streak formation in the chick embryo. Curr Top Dev Biol 2008;81:135-156.

-30 Mikawa T, Poh AM, Kelly KA, Ishii Y, Reese DE: Induction and patterning of the primitive streak, an organizing center of gastrulation in the amniote. Dev Dyn 2004;229:422-432.

31 Milbradt D: The acupuncture and oriental medicine news source: Bonghan channels in acupuncture: acupuncture today. 2009. http://acupuncturetoday.com/mpacms/at/article.php?id=31918 (accessed December 2012).

-32 Harrison LG: The Shaping of Life: The Generation of Biological Pattern. Cambridge, Cambridge University Press, 2011, p 206.

-33 Ereskovsky AV: The Comparative Embryology of Sponges. Dordrecht, Springer, 2010, p 236.

34 Catala M: Cell movements during gastrulation; in Tortori-Donati P, Rossi A (eds): Pediatric Neuroradiology: Brain. New York, Springer, 2005, p 1535.

-35 Chuai M, Zeng W, Yang X, Boychenko V, Glazier JA, Weijer CJ: Cell movement during chick primitive streak formation. Dev Biol 2006;296:137-149.

-36 Tam PP, Loebel DA: Gene function in mouse embryogenesis: get set for gastrulation. Nat Rev Genet 2007;8: 368-381. 